\title{
The suspension order of the real even dimensional projective space
}

\author{
By \\ Juno MukaI*
}

\begin{abstract}
The purpose of this paper is to prove the truth of the conjecture in [12]: The suspension order of the real even dimensional projective space coincides with its stable order determined by Toda [21] (see Silberbush and Ucci [19]). We obtain the assertion by proving that the suspension order of the real 6-projective space is 8 .
\end{abstract}

\section{Introduction}

In this paper all spaces, maps and homotopies are based. For a space $X$, we denote by $\Sigma X$ a suspension of $X$ and by $\iota_{X}$ the identity class of $X$. The order of $\iota_{\Sigma X} \in[\Sigma X, \Sigma X]$ is called the suspension order ([21]) or the characteristic [4] of $X$. The order of $\Sigma^{\infty} \iota_{X} \in\{X, X\}$ is called the stable order ([21]) of $X$. Let $\mathrm{P}^{n}$ be the real $n$-dimensional projective space. Adams [1, Theorem 7.4] showed that the $K O$-group $\widetilde{K O}\left(\mathrm{P}^{n}\right)$ is isomorphic to $\mathbb{Z}_{2^{\phi(n)}}$, where $\phi(n)$ is the number of integers $i$ satisfying $1 \leq i \leq n$ and $i \equiv 0,1,2$ or $4 \bmod 8$. Toda [21, Corollary 3 to Theorem 4.3] determined the stable order of $\mathrm{P}^{2 n}$ equal to $2^{\phi(2 n)}$. The purpose of the present paper is to show the following.

Theorem 1.1. The suspension order of $\mathrm{P}^{6}$ is 8 .

As an application of the theorem, we conclude that the suspension order of $\mathrm{P}^{2 n}$ coincides with its stable order (see [12, Appendix]). In other words, we obtain the following.

Corollary 1.2. The suspension order of $\mathrm{P}^{2 n}$ is $2^{\phi(2 n)}$.

For a space $X$ and its subspace $A$, let us denote by $i_{A, X}: A \rightarrow X$ the inclusion map and by $p_{X, A}: X \rightarrow X / A$ the map pinching $A$ to one point. We set $\mathrm{P}_{k}^{n}=\mathrm{P}^{n} / \mathrm{P}^{k-1}, i_{k, n}=i_{\mathrm{P}^{k}, \mathrm{P}^{n}}$ and $p_{n, k}=p_{\mathrm{P}^{n}, \mathrm{P}^{k}}$ for $k \leq n$. We set $\iota_{n}=\iota_{S^{n}}$. We denote by $\gamma_{n}: S^{n} \rightarrow \mathrm{P}^{n}$ the covering map.

2000 Mathematics Subject Classification(s). Primary 55P05, 55Q15; Secondary 55Q52 Received March 31, 2003

*Partially supported by Akio Satō, Shinshu University 101501 and Grant-in-Aid for Scientific Research (No. 15540067 (c), (2)), Japan Society for the Promotion of Science 
By abuse of notation, the same notation is often used for a mapping and its homotopy class.

Since $\pi_{n}\left(\mathrm{P}^{n+1}\right)=0$, we have

$$
i_{n, n+1} \circ \gamma_{n}=0 .
$$

From the cell structure of $\mathrm{P}^{n}$, we obtain

$$
p_{n, n-1} \circ \gamma_{n}=\left(1+(-1)^{n-1}\right) \iota_{n} .
$$

Let $\eta_{n} \in \pi_{n+1}\left(S^{n}\right)$ for $n \geq 2$ be the Hopf map and $\eta_{n}^{2}=\eta_{n} \circ \eta_{n+1} \in$ $\pi_{n+2}\left(S^{n}\right)$. We recall from [20] that

$$
\pi_{3}\left(S^{2}\right)=\mathbb{Z}\left\{\eta_{2}\right\}, \quad \pi_{n+1}\left(S^{n}\right)=\mathbb{Z}_{2}\left\{\eta_{n}\right\} \quad(n \geq 3)
$$

and

$$
\pi_{n+2}\left(S^{n}\right)=\mathbb{Z}_{2}\left\{\eta_{n}^{2}\right\} \quad(n \geq 2) .
$$

Here, for example, the notation $\pi_{n+1}\left(S^{n}\right)=\mathbb{Z}_{2}\left\{\eta_{n}\right\}$ indicates that $\pi_{n+1}\left(S^{n}\right)$ is isomorphic to $\mathbb{Z}_{2}$ and generated by $\eta_{n}$.

We set $M^{n}=\Sigma^{n-2} \mathrm{P}^{2}, i_{n}=\Sigma^{n-2} i_{1,2}$ and $p_{n}=\Sigma^{n-2} p_{2,1}$. Let $\tilde{\eta}_{2} \in \pi_{4}\left(M^{3}\right)$ be an element satisfying $p_{3} \tilde{\eta}_{2}=\eta_{3}([15$, Lemma 4.1]) and set

$$
\tilde{\eta}_{n}=\Sigma^{n-2} \tilde{\eta}_{2} \quad(n \geq 2) .
$$

Let $\bar{\eta}_{3} \in\left[M^{5}, S^{3}\right]$ be an extension of $\eta_{3}$ and set

$$
\bar{\eta}_{n}=\Sigma^{n-3} \bar{\eta}_{3} \quad(n \geq 3) .
$$

We obtain $\pi_{3}\left(M^{3}\right)=\mathbb{Z}_{4}\left\{i_{3} \eta_{2}\right\}$ and

$$
\pi_{n}\left(M^{n}\right)=\mathbb{Z}_{2}\left\{i_{n} \eta_{n-1}\right\} \quad(n \geq 4) .
$$

We recall an important relation ([3], [21])

$$
2 \iota_{M^{n}}=i_{n} \eta_{n-1} p_{n} \quad(n \geq 3) .
$$

We also obtain $\pi_{n+2}\left(M^{n+1}\right)=\mathbb{Z}_{4}\left\{\tilde{\eta}_{n}\right\}$,

$$
2 \tilde{\eta}_{n}=i_{n+1} \eta_{n}^{2} \quad(n \geq 2),
$$

$\left[M^{n+2}, S^{n}\right]=\mathbb{Z}_{4}\left\{\bar{\eta}_{n}\right\}$ and

$$
2 \bar{\eta}_{n}=\eta_{n}^{2} p_{n+2} \quad(n \geq 3) .
$$

Making use of the cofiber sequence

$$
(*)_{n} \quad S^{n} \stackrel{2 \iota_{n}}{\longrightarrow} S^{n} \stackrel{i_{n+1}}{\longrightarrow} M^{n+1} \stackrel{p_{n+1}}{\longrightarrow} S^{n+1} \longrightarrow \cdots
$$

and by the groups $\pi_{k}\left(M^{n}\right)$ for $k=n, n+1$, we get that (see [16, Lemma 1.5 (i)])

$$
\left[M^{n+1}, M^{n}\right]=\mathbb{Z}_{2}\left\{i_{n} \bar{\eta}_{n-1}\right\} \oplus \mathbb{Z}_{2}\left\{\tilde{\eta}_{n-1} p_{n+1}\right\} \quad(n \geq 4) .
$$

To prove Theorem 1.1, we need the following. 
Theorem 1.3. $2\left[M^{7}, \Sigma \mathrm{P}^{n}\right]=0$ for $n \geq 3$.

We note that $\mathrm{P}_{3}^{6}$ is identified with the mapping cone of $i_{4} \bar{\eta}_{3}$ :

$$
\mathrm{P}_{3}^{6}=M^{4} \cup_{i_{4} \bar{\eta}_{3}} C M^{5} .
$$

We set $i^{\prime}=i_{\mathrm{P}_{3}^{4}, \mathrm{P}_{3}^{6}}$ and $p^{\prime}=p_{\mathrm{P}_{3}^{6}, \mathrm{P}_{3}^{4}}$. We consider an element $\left(\Sigma i_{1,4}\right) \eta_{2} \bar{\eta}_{3} \in$ $\left[M^{5}, \Sigma \mathrm{P}^{4}\right]$. By [18, Lemma 5.2], we know a relation

$$
\left(\Sigma i_{2,4}\right) \tilde{\eta}_{2} \eta_{4}=0 \in \pi_{5}\left(\Sigma \mathrm{P}^{4}\right) .
$$

So, by (1.4) and (1.5),

$$
\begin{aligned}
\left(\Sigma i_{1,4}\right) \eta_{2} \bar{\eta}_{3} \circ i_{5} \bar{\eta}_{4} & =\left(\Sigma i_{1,4}\right) \eta_{2}^{2} \bar{\eta}_{4}=\left(\Sigma i_{2,4}\right) \tilde{\eta}_{2} \circ 2 \iota_{4} \circ \bar{\eta}_{4} \\
& =\left(\Sigma i_{2,4}\right) \tilde{\eta}_{2} \eta_{4}^{2} p_{6}=0 \in\left[M^{6}, \Sigma \mathrm{P}^{4}\right]
\end{aligned}
$$

and hence $\left(\Sigma i_{1,4}\right) \eta_{2} \bar{\eta}_{3}$ is extendible to $\overline{\left(\Sigma i_{1,4}\right) \eta_{2} \bar{\eta}_{3}} \in\left[\Sigma \mathrm{P}_{3}^{6}, \Sigma \mathrm{P}^{4}\right]$. By (1.3) and $(1.6)$

$$
i_{4} \bar{\eta}_{3} \circ i_{5} \eta_{4} p_{5}=2\left(i_{4} \bar{\eta}_{3}\right)=0 \in\left[M^{5}, M^{4}\right] .
$$

Therefore there exists a coextenstion $\widetilde{i_{5} \eta_{4} p_{5}} \in\left[M^{6}, \mathrm{P}_{3}^{6}\right]$ of $i_{5} \eta_{4} p_{5}$ satisfying $p_{*}^{\prime} \widetilde{i_{5} \eta_{4} p_{5}}=i_{6} \eta_{5} p_{6}$. The Toda bracket

$$
\left\{\left(\Sigma i_{1,4}\right) \eta_{2} \bar{\eta}_{3}, i_{5} \bar{\eta}_{4}, i_{6} \eta_{5} p_{6}\right\}_{1} \subset\left[M^{7}, \Sigma \mathrm{P}^{4}\right]
$$

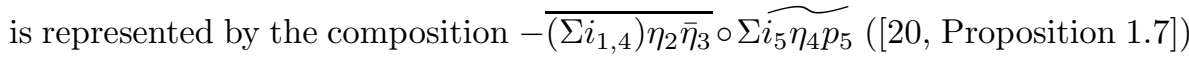
as shown in the commutative diagram $\left(\xi=\left(\Sigma i_{1,4}\right) \eta_{2} \bar{\eta}_{3}\right)$ :

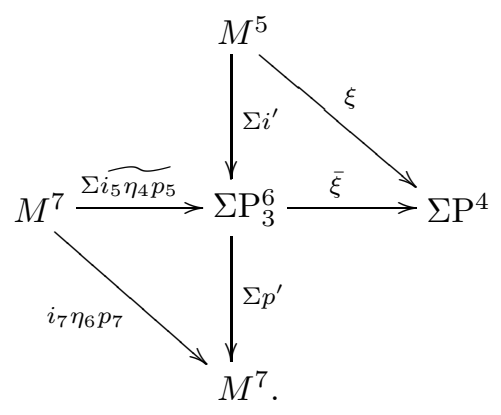

The key to proving Theorem 1.1 is to find out the following.

\section{Theorem 1.4.}

(i) $4 \iota_{\Sigma \mathrm{P}^{6}} \equiv\left(\Sigma i_{4,6}\right) \overline{\left(\Sigma i_{1,4}\right) \eta_{2} \bar{\eta}_{3}} \circ \Sigma p_{6,2} \bmod \left[M^{7}, \Sigma \mathrm{P}^{6}\right] \circ \Sigma p_{6,4}$.

(ii) $\left\{\left(\Sigma i_{1,4}\right) \eta_{2} \bar{\eta}_{3}, i_{5} \bar{\eta}_{4}, i_{6} \eta_{5} p_{6}\right\}_{1}$ consists of a single element and $\overline{2\left(\Sigma i_{1,4}\right) \eta_{2} \bar{\eta}_{3}}$ $=\left\{\left(\Sigma i_{1,4}\right) \eta_{2} \bar{\eta}_{3}, i_{5} \bar{\eta}_{4}, i_{6} \eta_{5} p_{6}\right\}_{1} \circ \Sigma p^{\prime} \in\left[\Sigma \mathrm{P}_{3}^{6}, \Sigma \mathrm{P}^{4}\right]$.

(iii) $\left(\Sigma \gamma_{5}\right) \eta_{6} p_{7}=\left(\Sigma i_{4,5}\right) \circ\left\{\left(\Sigma i_{1,4}\right) \eta_{2} \bar{\eta}_{3}, i_{5} \bar{\eta}_{4}, i_{6} \eta_{5} p_{6}\right\}_{1} \in\left[M^{7}, \Sigma \mathrm{P}^{5}\right]$. 
Theorem 1.1 is a direct consequence of Theorems 1.3, 1.4 and (1.1).

We use the composition methods in [20] arranged for suspended real projective spaces. And we are based on the result in [18]. The exact sequence of James [10, Theorem 2.1] is used to determine the group structure of $\pi_{6}\left(\Sigma \mathrm{P}^{2} \wedge \mathrm{P}^{2}\right)$ (Lemma 3.1 (ii)). To prove Theorem 1.3, it is essential to find out the triviality of some element of $\left[M^{7}, \Sigma \mathrm{P}^{2} \wedge \mathrm{P}^{2}\right](3.3)$ by considering the Whitehead product $\left[\iota_{M^{4}}, 2 \iota_{M^{4}}\right]$.

\section{Recollection of known results}

Let $\nu^{\prime}$ be a generator of the 2-primary component $\pi_{6}^{3} \cong \mathbb{Z}_{4}$ of $\pi_{6}\left(S^{3}\right)$. We need the following facts [20]:

$$
\begin{gathered}
\pm \nu^{\prime}=\bar{\eta}_{3} \tilde{\eta}_{4}, \\
2 \nu^{\prime}=\eta_{3}^{3}, \eta_{3} \nu_{4}=\nu^{\prime} \eta_{6}, \\
\pi_{7}^{4}=\mathbb{Z}\left\{\nu_{4}\right\} \oplus \mathbb{Z}_{4}\left\{\Sigma \nu^{\prime}\right\}
\end{gathered}
$$

and

$$
\pm\left[\iota_{4}, \iota_{4}\right]=2 \nu_{4}-\Sigma \nu^{\prime} .
$$

We also recall from [13], [14], [15], [16] that

$$
\begin{aligned}
\pi_{7}\left(M^{5}\right) & =\mathbb{Z}_{4}\left\{i_{5} \nu_{4}\right\} \oplus \mathbb{Z}_{2}\left\{\tilde{\eta}_{4} \eta_{6}\right\}, \\
\pi_{6}\left(M^{4}\right) & =\mathbb{Z}_{4}\{\delta\} \oplus \mathbb{Z}_{2}\left\{\tilde{\eta}_{3} \eta_{5}\right\}, \\
2 \delta & =i_{4} \nu^{\prime}, \\
\Sigma \delta & =2\left(i_{5} \nu_{4}\right) \in \pi_{7}\left(M^{5}\right), \\
\pi_{7}\left(M^{4}\right) & =\mathbb{Z}_{2}\left\{\delta \eta_{6}\right\} \oplus \mathbb{Z}_{2}\left\{\tilde{\eta}_{3} \eta_{5}^{2}\right\}
\end{aligned}
$$

and

$$
\left[\iota_{M^{4}}, i_{4}\right]=\delta p_{6}
$$

Making use of the cofiber sequence $(*)_{k}$ for $k=5,6$, by the group structures $\pi_{k}\left(M^{5}\right)(k=6,7), \pi_{k}^{3}(k=5,6), \pi_{k}\left(M^{4}\right)(5 \leq k \leq 7)$ and by (1.4), (2.2), (1.3), we obtain the following (see $[16$, Lemma 1.5 (iii)]).

\section{Lemma 2.1.}

(i) $\left[M^{7}, M^{5}\right]=\mathbb{Z}_{2}\left\{i_{5} \eta_{4} \bar{\eta}_{5}\right\} \oplus \mathbb{Z}_{2}\left\{\tilde{\eta}_{4} \eta_{6} p_{7}\right\} \oplus \mathbb{Z}_{2}\left\{i_{5} \nu_{4} p_{7}\right\}$.

(ii) $\left[M^{6}, S^{3}\right]=\mathbb{Z}_{2}\left\{\eta_{3} \bar{\eta}_{4}\right\} \oplus \mathbb{Z}_{2}\left\{\nu^{\prime} p_{6}\right\}$.

(iii) $\left[M^{6}, M^{4}\right]=\mathbb{Z}_{2}\left\{i_{4} \eta_{3} \bar{\eta}_{4}\right\} \oplus \mathbb{Z}_{2}\left\{\tilde{\eta}_{3} \eta_{5} p_{6}\right\} \oplus \mathbb{Z}_{2}\left\{\delta p_{6}\right\}$.

(iv) $\left[M^{7}, M^{4}\right]=\mathbb{Z}_{4}\left\{\tilde{\eta}_{3} \bar{\eta}_{5}\right\} \oplus \mathbb{Z}_{2}\left\{\overline{u_{4} \nu^{\prime}}\right\} \oplus \mathbb{Z}_{2}\left\{\delta \eta_{6} p_{7}\right\}$, where $2 \tilde{\eta}_{3} \bar{\eta}_{5}=\tilde{\eta}_{3} \eta_{5}^{2} p_{7}$ and $\overline{\imath_{4} \nu^{\prime}}$ is an extension of $i_{4} \nu^{\prime}$.

The smash product of $\mathrm{P}^{2}$ with itself has the following cell structure:

$$
\mathrm{P}^{2} \wedge \mathrm{P}^{2}=M^{3} \cup_{2 \iota_{M^{3}}} C M^{3},
$$


where we take $M^{3}=\mathrm{P}^{2} \wedge S^{1}$ and $2 \iota_{M^{3}}=\iota_{\mathrm{P}^{2}} \wedge 2 \iota_{1}$. So, by (1.3), it turns to the form:

$$
\mathrm{P}^{2} \wedge \mathrm{P}^{2}=\left(M^{3} \vee S^{3}\right) \cup_{f_{1}^{\prime} i_{3} \eta_{2}+2 f_{2}^{\prime}} e^{4},
$$

where $f_{1}^{\prime}: M^{3} \rightarrow M^{3} \vee S^{3}$ and $f_{2}^{\prime}: S^{3} \rightarrow M^{3} \vee S^{3}$ be the embeddings to the first

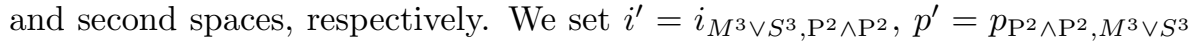
and $p^{\prime \prime}=p_{\mathrm{P}^{2} \wedge \mathrm{P}^{2}, M^{3}}$. Then we can take $i^{\prime} f_{1}^{\prime}=\iota_{\mathrm{P}^{2}} \wedge i_{2}$. Since $p^{\prime \prime} \circ i^{\prime} f_{2}^{\prime}=i_{4}, i^{\prime} f_{2}^{\prime}$ is a coextension of $i_{3}$. We set $\tilde{\imath}_{3}=i^{\prime} f_{2}^{\prime}, \tilde{\imath}_{n}=\Sigma^{n-3} \tilde{\imath}_{3}(n \geq 3), f_{k}=\Sigma f_{k}^{\prime}(k=1,2)$, $i=\Sigma i^{\prime}$ and $p=\Sigma p^{\prime}$. By [17, Lemma 2.4],

$$
\pi_{3}\left(\mathrm{P}^{2} \wedge \mathrm{P}^{2}\right)=\mathbb{Z}_{8}\left\{\tilde{\imath}_{3}\right\}
$$

and

$$
\pi_{n}\left(\Sigma^{n-3}\left(\mathrm{P}^{2} \wedge \mathrm{P}^{2}\right)\right)=\mathbb{Z}_{4}\left\{\tilde{\imath}_{n}\right\} \quad(n \geq 4),
$$

where

$$
2 \tilde{\imath}_{n}=\left(\Sigma^{n-3}\left(i_{2} \wedge i_{2}\right)\right) \eta_{n-1} \quad(n \geq 3) .
$$

Let us recall that $\mathrm{P}^{3}$ is homeomorphic to the 3-rd rotation group. Let $h: \Sigma \mathrm{P}^{3} \wedge \mathrm{P}^{3} \rightarrow \Sigma \mathrm{P}^{3}$ be the Hopf construction induced from the multiplication of the topological group $\mathrm{P}^{3}$. We know the following ([5], [11]).

Lemma 2.2. $\quad$ There exists a direct sum decomposition for a space $X$ :

$$
\left[\Sigma^{2} X, \Sigma \mathrm{P}^{3}\right]=h_{*}\left[\Sigma^{2} X, \Sigma \mathrm{P}^{3} \wedge \mathrm{P}^{3}\right] \oplus \Sigma\left[\Sigma X, \mathrm{P}^{3}\right] .
$$

The following is [18, Lemma 3.2].

Lemma 2.3. $\quad \Sigma \mathrm{P}^{3} \wedge \mathrm{P}^{3}=\left(\left(\Sigma \mathrm{P}^{2} \wedge \mathrm{P}^{2}\right) \cup_{i \nu^{\prime}} e^{7}\right) \vee M^{6} \vee M^{6}$, where $i=i_{3} \wedge i_{2}$.

Let $h_{0}=\left.h\right|_{\left(\Sigma \mathrm{P}^{2} \wedge \mathrm{P}^{2}\right) \cup_{i \nu^{\prime}} e^{7}}, h^{\prime}=\left.h\right|_{\Sigma \mathrm{P}^{2} \wedge \mathrm{P}^{2}}, h^{\prime \prime}=\left.h\right|_{M^{4}}$ and $h^{\prime \prime \prime}=\left.h\right|_{S^{3}}$ be the restrictions of $h$, respectively. By [18, Lemma 2.3 (i)],

$$
h^{\prime \prime \prime}=\left(\Sigma i_{1,3}\right) \eta_{2} \in \pi_{3}\left(\Sigma \mathrm{P}^{3}\right)
$$

and

$$
h^{\prime} \tilde{\imath}_{4}= \pm\left(\Sigma i_{2,3}\right) \tilde{\eta}_{2} \in \pi_{4}\left(\Sigma \mathrm{P}^{3}\right) .
$$

By [18, Lemma 5.2, (6.3)],

$$
\Sigma \gamma_{4}=\left(\Sigma i_{3,4}\right) h^{\prime \prime} \tilde{\eta}_{3} \in \pi_{5}\left(\Sigma \mathrm{P}^{4}\right)
$$

and

$$
4 \iota_{\Sigma \mathrm{P}^{4}}=\left(\Sigma i_{1,4}\right) \eta_{2} \bar{\eta}_{3} \Sigma p_{4,2} .
$$

3. Proof of the fact that $2\left[M^{7}, \Sigma \mathrm{P}^{3}\right]=0$

First we show the following. 


\section{Lemma 3.1.}

(i) $\pi_{5}\left(\Sigma \mathrm{P}^{2} \wedge \mathrm{P}^{2}\right)=\mathbb{Z}_{2}\left\{\left(\iota_{M^{3}} \wedge i_{2}\right) \tilde{\eta}_{3}\right\} \oplus \mathbb{Z}_{2}\left\{\tilde{\imath}_{4} \eta_{4}\right\}$ and $\Sigma^{n}: \pi_{5}\left(\Sigma \mathrm{P}^{2} \wedge \mathrm{P}^{2}\right)$ $\rightarrow \pi_{n+5}\left(\sum^{n+1} \mathrm{P}^{2} \wedge \mathrm{P}^{2}\right)$ is an isomorphism for $n \geq 1$.

(ii) $\pi_{6}\left(\Sigma \mathrm{P}^{2} \wedge \mathrm{P}^{2}\right)=\mathbb{Z}_{4}\left\{\left(\iota_{M^{3}} \wedge i_{2}\right) \delta\right\} \oplus \mathbb{Z}_{2}\left\{\left(\iota_{M^{3}} \wedge i_{2}\right) \tilde{\eta}_{3} \eta_{5}\right\} \oplus \mathbb{Z}_{2}\left\{\tilde{\imath}_{4} \eta_{4}^{2}\right\} \oplus$ $\mathbb{Z}_{2}\left\{\left[i_{3} \wedge i_{2}, \tilde{\imath}_{4}\right]\right\}$, where $2\left(\left(\iota_{M^{3}} \wedge i_{2}\right) \delta\right)=\left(i_{3} \wedge i_{2}\right) \nu^{\prime}$.

Proof. (i) is easily obtained (see $[18, \S 4])$.

The relation in (ii) is obtained from (2.2). We consider the homotopy exact sequence of a pair $\left(\Sigma \mathrm{P}^{2} \wedge \mathrm{P}^{2}, M^{4} \vee S^{4}\right)$ :

$$
\begin{aligned}
& \pi_{7}\left(\Sigma \mathrm{P}^{2} \wedge \mathrm{P}^{2}, M^{4} \vee S^{4}\right) \stackrel{\partial}{\longrightarrow} \pi_{6}\left(M^{4} \vee S^{4}\right) \stackrel{i_{*}}{\longrightarrow} \pi_{6}\left(\Sigma \mathrm{P}^{2} \wedge \mathrm{P}^{2}\right) \\
& \stackrel{j_{*}}{\longrightarrow} \pi_{6}\left(\Sigma \mathrm{P}^{2} \wedge \mathrm{P}^{2}, M^{4} \vee S^{4}\right) \stackrel{\partial}{\longrightarrow} \cdots .
\end{aligned}
$$

By Blakers-Massey [7], $\pi_{6}\left(\Sigma \mathrm{P}^{2} \wedge \mathrm{P}^{2}, M^{4} \vee S^{4}\right) \cong \pi_{6}\left(S^{5}\right)$. The generator of the relative homotopy group is denoted by $\hat{\eta}_{4}$, satisfying $p_{*} \hat{\eta}_{4}=\eta_{5}$. We have $\pi_{5}\left(M^{4} \vee S^{4}\right) \cong \mathbb{Z}_{4} \oplus \mathbb{Z}_{2}$. So, by (1.4),

$$
\partial \hat{\eta}_{4}=\left(f_{1} i_{4} \eta_{3}+2 f_{2}\right) \circ \eta_{4}=f_{1} i_{4} \eta_{3}^{2}=2 f_{1} \tilde{\eta}_{3} \neq 0 .
$$

Hence $i_{*}: \pi_{6}\left(M^{4} \vee S^{4}\right) \rightarrow \pi_{6}\left(\Sigma \mathrm{P}^{2} \wedge \mathrm{P}^{2}\right)$ is an epimorphism.

Since $\pi_{7}\left(M^{4} \times S^{4}, M^{4} \vee S^{4}\right) \cong \pi_{7}\left(M^{8}\right), M^{4} \times S^{4}=\left(M^{4} \vee S^{4}\right) \cup_{\left[f_{1}, f_{2}\right]} C M^{7}$ and $\left[f_{1}, f_{2}\right] \circ i_{7}=\left[f_{1} i_{4}, f_{2}\right]$, we obtain

$$
\pi_{6}\left(M^{4} \vee S^{4}\right)=\mathbb{Z}_{4}\left\{f_{1} \delta\right\} \oplus \mathbb{Z}_{2}\left\{f_{1} \tilde{\eta}_{3} \eta_{5}\right\} \oplus \mathbb{Z}_{2}\left\{f_{2} \eta_{4}^{2}\right\} \oplus \mathbb{Z}_{2}\left\{\left[f_{1} i_{4}, f_{2}\right]\right\} .
$$

By [10, Theorem 2.1], $\pi_{7}\left(\Sigma \mathrm{P}^{2} \wedge \mathrm{P}^{2}, M^{4} \vee S^{4}\right)=\mathbb{Z}_{2}\left\{\widehat{\eta_{4}^{2}}\right\} \oplus \mathbb{Z}_{2}\left\{\left[\omega, f_{1} i_{4}\right]\right\}$, where $\omega$ is the characteristic map of the 5-cell of $\Sigma \mathrm{P}^{2} \wedge \mathrm{P}^{2},[$,$] stands for the relative$ Whitehead product $([8])$ and $\widehat{\eta_{4}^{2}}$ is an element satisfying $p_{*} \widehat{\eta_{4}^{2}}=\eta_{5}^{2}$. We have $\partial\left(\widehat{\eta_{4}^{2}}\right)=f_{1} i_{4} \eta_{3}^{3}=0$. By $[8]$ and the fact that $\left[\eta_{3}, \iota_{3}\right]=0$, we see that

$$
\partial\left[\omega, f_{1} i_{4}\right]=-\left[f_{1} i_{4} \eta_{3}+2 f_{2}, f_{1} i_{4}\right]=\left(f_{1} i_{4}\right)\left[\eta_{3}, \iota_{3}\right]+2\left[f_{2}, f_{1} i_{4}\right]=0 .
$$

This leads to (ii), completing the proof.

By use of [2, Theorem 2.4] and [6, Proposition II. 3.2], we obtain the following (see [15, Remark, p. 273]).

Lemma 3.2. Let $\alpha \in[\Sigma A, X], \beta \in[\Sigma B, X], \delta \in[D, A]$ and $\varepsilon \in[E, B]$, where $A, B, D, E$ are polyhedra and $X$ is a space. Then

$$
[\alpha \circ \Sigma \delta, \beta \circ \Sigma \varepsilon]=[\alpha, \beta] \circ \Sigma(\delta \wedge \varepsilon) .
$$

Next we show the following.

Lemma 3.3. $2\left[M^{7}, \Sigma \mathrm{P}^{2} \wedge \mathrm{P}^{2}\right]=0$. 
Proof. Since $\left[\iota_{M^{3}} \wedge i_{2}, \tilde{r}_{4}\right] \circ \Sigma\left(\left(i_{2} \wedge \iota_{1}\right) \wedge \iota_{3}\right)=\left[i_{3} \wedge i_{2}, \tilde{r}_{4}\right]$ by Lemma 3.2, $\left[\iota_{M^{3}} \wedge i_{2}, \tilde{\imath}_{4}\right]$ is an extension of $\left[i_{3} \wedge i_{2}, \tilde{\imath}_{4}\right]$. By use of $(*)_{6}$ and by Lemma 3.1 (ii), we obtain

$$
\begin{aligned}
{\left[M^{7}, \Sigma \mathrm{P}^{2} \wedge \mathrm{P}^{2}\right]=\{} & \left(\iota_{M^{3}} \wedge i_{2}\right) \overline{\imath_{4} \nu^{\prime}},\left(\iota_{M^{3}} \wedge i_{2}\right) \tilde{\eta}_{3} \bar{\eta}_{5}, \tilde{\imath}_{4} \eta_{4} \bar{\eta}_{5}, \\
& {\left.\left[\iota_{M^{3}} \wedge i_{2}, \tilde{\imath}_{4}\right]\right\}+\pi_{7}\left(\Sigma \mathrm{P}^{2} \wedge \mathrm{P}^{2}\right) \circ p_{7} . }
\end{aligned}
$$

We have $2\left(\eta_{4} \bar{\eta}_{5}\right)=0$ and $2 \overline{\imath_{4} \nu^{\prime}}=0$ by Lemma 2.1 (iv). By the relation $2 i_{2}=0$,

$$
2\left[\iota_{M^{3}} \wedge i_{2}, \tilde{\imath}_{4}\right]=\left[\iota_{M^{3}} \wedge i_{2}, \tilde{\imath}_{4}\right] \circ \Sigma\left(\left(i_{2} \wedge 2 \iota_{1}\right) \wedge \iota_{3}\right)=0
$$

and

$$
2\left(\left(\iota_{M^{3}} \wedge i_{2}\right) \tilde{\eta}_{3} \bar{\eta}_{5}\right)=0 .
$$

Hence, by the relation $2 p_{7}=0$, the assertion follows.

We examine the Whitehead product $\left[\iota_{M^{4}}, 2 \iota_{M^{4}}\right]$. By (1.3), Lemma 3.2, (2.3) and by the fact that

$$
\iota_{\mathrm{P}^{2}} \wedge \eta_{2}=i_{4} \bar{\eta}_{3}+\tilde{\eta}_{3} p_{5} \in\left[M^{5}, M^{4}\right]
$$

we get the following in $\left[\Sigma M^{3} \wedge M^{3}, M^{4}\right]$ :

$$
\begin{aligned}
{\left[\iota_{M^{4}}, 2 \iota_{M^{4}}\right]=\left[\iota_{M^{4}}, i_{4} \eta_{3} p_{4}\right] } & =\left[\iota_{M^{4}}, i_{4}\right] \circ \Sigma\left(\iota_{M^{3}} \wedge\left(\eta_{2} p_{3}\right)\right) \\
& =\left[\iota_{M^{4}}, i_{4}\right] \circ \Sigma\left(\iota_{M^{3}} \wedge \eta_{2}\right) \circ \Sigma\left(\iota_{M^{3}} \wedge p_{3}\right) \\
& =\delta \eta_{6} \circ p_{7} \circ \Sigma\left(\iota_{M^{3}} \wedge p_{3}\right) .
\end{aligned}
$$

Since

$$
p_{7} \circ \Sigma\left(\iota_{M^{3}} \wedge p_{3}\right)=\Sigma\left(p_{3} \wedge p_{3}\right) \in\left[\Sigma M^{3} \wedge M^{3}, S^{7}\right],
$$

we obtain

$$
\left[\iota_{M^{4}}, 2 \iota_{M^{4}}\right]=\delta \eta_{6} \circ \Sigma\left(p_{3} \wedge p_{3}\right) \in\left[\Sigma M^{3} \wedge M^{3}, M^{4}\right] .
$$

Now we show the following.

Lemma 3.4. $2\left[M^{7},\left(\Sigma \mathrm{P}^{2} \wedge \mathrm{P}^{2}\right) \cup_{i \nu^{\prime}} e^{7}\right]=0$.

Proof. By (3.2) and the fact that $2\left(\iota_{M^{3}} \wedge i_{2}\right)=0$,

$$
\begin{aligned}
\left(\iota_{M^{3}} \wedge i_{2}\right) \delta \eta_{6} \circ \Sigma\left(p_{3} \wedge p_{3}\right) & =\left[\iota_{M^{3}} \wedge i_{2}, 2\left(\iota_{M^{3}} \wedge i_{2}\right)\right] \\
& =0 \in\left[\Sigma M^{3} \wedge M^{3}, \Sigma \mathrm{P}^{2} \wedge \mathrm{P}^{2}\right] .
\end{aligned}
$$

So, by making use of the cofiber sequence

$$
M^{6} \stackrel{\iota^{M^{4}} \wedge i_{3}}{\longrightarrow} \Sigma M^{3} \wedge M^{3} \stackrel{\iota_{M^{4}} \wedge p_{3}}{\longrightarrow} M^{7} \stackrel{2 \iota_{M^{7}}}{\longrightarrow} M^{7} \longrightarrow \cdots
$$


and by (3.1), we obtain $\left(\iota_{M^{3}} \wedge i_{2}\right) \delta \eta_{6} p_{7} \in 2\left[M^{7}, \Sigma \mathrm{P}^{2} \wedge \mathrm{P}^{2}\right]$. Hence, by Lemma 3.3 ,

$$
\left(\iota_{M^{3}} \wedge i_{2}\right) \delta \eta_{6} p_{7}=0 \in\left[M^{7}, \Sigma \mathrm{P}^{2} \wedge \mathrm{P}^{2}\right] .
$$

We set $B=\left(\Sigma \mathrm{P}^{2} \wedge \mathrm{P}^{2}\right) \cup_{i \nu^{\prime}} e^{7}$ and $r=i_{\Sigma \mathrm{P}^{2} \wedge \mathrm{P}^{2}, B}$. From the homotopy exact sequence of a pair $\left(B, \Sigma \mathrm{P}^{2} \wedge \mathrm{P}^{2}\right)$ and by Lemma 3.1 (ii), we obtain

$$
\pi_{6}(B)=\left\{r\left(\iota_{M^{3}} \wedge i_{2}\right) \delta, r\left(\iota_{M^{3}} \wedge i_{2}\right) \tilde{\eta}_{3} \eta_{5}, r \tilde{\imath}_{4} \eta_{4}^{2}, r\left[i_{3} \wedge i_{2}, \tilde{\imath}_{4}\right]\right\} \cong\left(\mathbb{Z}_{2}\right)^{4} .
$$

So, by use of $(*)_{6}$, we obtain

$$
\left[M^{7}, B\right]=\left\{\overline{r\left(\iota_{M^{3}} \wedge i_{2}\right) \delta}, r\left(\iota_{M^{3}} \wedge i_{2}\right) \tilde{\eta}_{3} \bar{\eta}_{5}, r \tilde{\imath}_{4} \eta_{4} \bar{\eta}_{5}, r\left[\iota_{M^{3}} \wedge i_{2}, \tilde{\imath}_{4}\right]\right\}+\pi_{7}(B) \circ p_{7},
$$

and hence, $2\left[M^{7}, B\right]=\left\{2 \overline{r\left(\iota_{M^{3}} \wedge i_{2}\right) \delta}\right\}$. By (1.3) and (3.3),

$$
\overline{2 r\left(\iota_{M^{3}} \wedge i_{2}\right) \delta}=r\left(\iota_{M^{3}} \wedge i_{2}\right) \delta \eta_{6} p_{7}=0 .
$$

This leads to the assertion, completing the proof.

Since $r \circ\left(i_{3} \wedge i_{2}\right) \nu^{\prime}=0$ and $h^{\prime \prime \prime} \nu^{\prime}=h_{0} \circ r\left(i_{3} \wedge i_{2}\right) \nu^{\prime}$, we obtain

$$
\left(\Sigma i_{1,3}\right) \eta_{2} \nu^{\prime}=h^{\prime \prime \prime} \nu^{\prime}=0 \in \pi_{6}\left(\Sigma \mathrm{P}^{3}\right) .
$$

By Lemma 2.3, we have

$$
\pi_{6}\left(\Sigma \mathrm{P}^{3} \wedge \mathrm{P}^{3}\right) \cong \pi_{6}(B) \oplus \pi_{6}\left(M^{6}\right) \oplus \pi_{6}\left(M^{6}\right) .
$$

Since $2 \pi_{6}(B)=2 \pi_{6}\left(M^{6}\right)=0$, we get that $2 \pi_{6}\left(\Sigma \mathrm{P}^{3} \wedge \mathrm{P}^{3}\right)=0$. We have $2 \Sigma \pi_{5}\left(\mathrm{P}^{3}\right)=0$. So, by Lemma 2.2 , we obtain the following.

Lemma 3.5. $\quad 2 \pi_{6}\left(\Sigma \mathrm{P}^{3}\right)=0$.

We show the following.

Lemma 3.6. $\left(\Sigma i_{3, n}\right)_{*}: \pi_{6}\left(\Sigma \mathrm{P}^{3}\right) \rightarrow \pi_{6}\left(\Sigma \mathrm{P}^{n}\right)$ is an epimorphism for $n=4, n \geq 6$ and $\pi_{6}\left(\Sigma \mathrm{P}^{5}\right)=\mathbb{Z}\left\{\Sigma \gamma_{5}\right\} \oplus\left(\Sigma i_{3,5}\right)_{*} \pi_{6}\left(\Sigma \mathrm{P}^{3}\right)$.

Proof. In the homotopy exact sequence of a pair $\left(\Sigma \mathrm{P}^{4}, \Sigma \mathrm{P}^{3}\right)$, the connecting homomorphism $\partial: \pi_{6}\left(\Sigma \mathrm{P}^{4}, \Sigma \mathrm{P}^{3}\right) \rightarrow \pi_{5}\left(\Sigma \mathrm{P}^{3}\right)$ is a monomorphism by $\left[18\right.$, Theorem 5.3] and its proof. Hence $\left(\Sigma i_{3,4}\right)_{*}: \pi_{6}\left(\Sigma \mathrm{P}^{3}\right) \rightarrow \pi_{6}\left(\Sigma \mathrm{P}^{4}\right)$ is an epimorphism.

By making use of the homotopy exact sequence of a pair $\left(\Sigma \mathrm{P}^{5}, \Sigma \mathrm{P}^{4}\right)$ and by (1.2), we conclude that

$$
\pi_{6}\left(\Sigma \mathrm{P}^{5}\right)=\mathbb{Z}\left\{\Sigma \gamma_{5}\right\} \oplus\left(\Sigma i_{4,5}\right)_{*} \pi_{6}\left(\Sigma \mathrm{P}^{4}\right) .
$$

Obviously $\left(\Sigma i_{5, n}\right)_{*}: \pi_{6}\left(\Sigma \mathrm{P}^{5}\right) \rightarrow \pi_{6}\left(\Sigma \mathrm{P}^{n}\right)$ for $n \geq 6$ is an epimorphism. This completes the proof. 
Now we prove Theorem 1.3.

Proof of Theorem 1.3.

By Lemma 3.5, any element $\alpha \in \pi_{6}\left(\Sigma \mathrm{P}^{3}\right)$ is extendible to $\bar{\alpha} \in\left[M^{7}, \Sigma \mathrm{P}^{3}\right]$. So, by use of $(*)_{6}$ and by Lemma 3.6 , we obatin

$$
\left[M^{7}, \Sigma \mathrm{P}^{n}\right]=\left\{\left(\Sigma i_{3, n}\right) \bar{\alpha}\right\}+\pi_{7}\left(\Sigma \mathrm{P}^{n}\right) \circ p_{7}\left(\alpha \in \pi_{6}\left(\Sigma \mathrm{P}^{3}\right)\right) .
$$

Therefore it suffices to prove that $2\left[M^{7}, \Sigma \mathrm{P}^{3}\right]=0$. By [7], $\left[C M^{7}, M^{7} ; B \times\right.$ $\left.M^{6}, B \vee M^{6}\right] \cong\left[M^{8}, B \wedge M^{6}\right]\left(B=\left(\Sigma \mathrm{P}^{2} \wedge \mathrm{P}^{2}\right) \cup_{i \nu^{\prime}} e^{7}\right)$. So, by Lemma 2.3, we have

$$
\begin{aligned}
& {\left[M^{7}, \Sigma \mathrm{P}^{3} \wedge \mathrm{P}^{3}\right]} \\
& \quad \cong\left[M^{7}, B\right] \oplus\left[M^{7}, M^{6}\right] \oplus\left[M^{7}, M^{6}\right] \oplus\left[M^{8}, B \wedge M^{6}\right] \oplus\left[M^{8}, B \wedge M^{6}\right] .
\end{aligned}
$$

By Lemma $3.4,2\left[M^{7}, B\right]=0$. By (1.6), $2\left[M^{7}, M^{6}\right]=0$. Since $\left[M^{8}, B \wedge\right.$ $\left.M^{6}\right] \cong\left[M^{8}, M^{9}\right] \cong \mathbb{Z}_{2}$, we obtain $2\left[M^{8}, B \wedge M^{6}\right]=0$. By Lemma 2.1 (ii), $\left[M^{6}, \mathrm{P}^{3}\right] \cong\left[M^{6}, S^{3}\right] \cong\left(\mathbb{Z}_{2}\right)^{2}$. This shows that $2 \Sigma\left[M^{6}, \mathrm{P}^{3}\right]=0$. Hence, by Lemma 2.2, we conclude that $2\left[M^{7}, \Sigma \mathrm{P}^{3}\right]=0$. Thus the proof of Theorem 1.3 is complete.

We obtain the following.

Corollary 3.7. $\quad \pi_{6}\left(\Sigma \mathrm{P}^{n}\right) \circ \eta_{6} p_{7}=0$ for $n \geq 3, n \neq 5$ and $\pi_{6}\left(\Sigma \mathrm{P}^{5}\right) \circ$ $\eta_{6} p_{7}=\left\{\left(\Sigma \gamma_{5}\right) \eta_{6} p_{7}\right\}$.

Proof. Let $\alpha \in \pi_{6}\left(\Sigma \mathrm{P}^{n}\right)$ for $n \geq 3$ be a nontrivial element except for $\Sigma \gamma_{5}$. Then, by Lemmas 3.5 and 3.6, the order of $\alpha$ is 2 , and hence $\alpha$ is extendible to $\bar{\alpha} \in\left[M^{7}, \Sigma \mathrm{P}^{n}\right]$. Thus, by (1.3) and Theorem 1.3, $\alpha \circ\left(\eta_{6} p_{7}\right)=$ $2 \bar{\alpha} \in 2\left[M^{7}, \Sigma \mathrm{P}^{n}\right]=0$. This completes the proof.

\section{Proof of Theorem 1.1}

First of all we show the following.

\section{Lemma 4.1.}

(i) $\Sigma\left[M^{6}, M^{4}\right]=\mathbb{Z}_{2}\left\{i_{5} \eta_{4} \bar{\eta}_{5}\right\} \oplus \mathbb{Z}_{2}\left\{\tilde{\eta}_{4} \eta_{6} p_{7}\right\}$.

(ii) $\Sigma\left[M^{6}, M^{3}\right]=\mathbb{Z}_{4}\left\{\tilde{\eta}_{3} \bar{\eta}_{5}\right\}$.

Proof. By the fact that $\Sigma\left(\delta p_{6}\right)=\Sigma\left[\iota_{M^{4}}, i_{4}\right]=0$ (2.3), (i) is a direct consequence of Lemma 2.1 (iii).

We know that $\tilde{\eta}_{3} \bar{\eta}_{5} \in \Sigma\left[M^{6}, M^{3}\right]$. We consider the Hopf homomorphism $H:\left[M^{7}, M^{4}\right] \rightarrow\left[M^{7}, \Sigma M^{3} \wedge M^{3}\right]$. By Lemma 3.1 (i),

$$
\pi_{7}\left(\Sigma M^{3} \wedge M^{3}\right)=\mathbb{Z}_{2}\left\{\tilde{\imath}_{6} \eta_{6}\right\} \oplus \mathbb{Z}_{2}\left\{\left(\iota_{M^{4}} \wedge i_{3}\right) \tilde{\eta}_{5}\right\} .
$$

So, by use of $(*)_{6}$ combining with the fact that $\pi_{6}\left(\Sigma M^{3} \wedge M^{3}\right)=\mathbb{Z}_{4}\left\{\tilde{\imath}_{6}\right\}$ and $2 \tilde{\imath}_{6}=\left(i_{4} \wedge i_{3}\right) \eta_{5}(2.4)$, we obtain

$$
\left[M^{7}, \Sigma M^{3} \wedge M^{3}\right]=\mathbb{Z}_{2}\left\{\left(i_{4} \wedge i_{3}\right) \bar{\eta}_{5}\right\} \oplus \mathbb{Z}_{2}\left\{\tilde{\imath}_{6} \eta_{6} p_{7}\right\} \oplus \mathbb{Z}_{2}\left\{\left(\iota_{M^{4}} \wedge i_{3}\right) \tilde{\eta}_{5} p_{7}\right\} .
$$


By [14, Proposition 14],

$$
H(\delta)= \pm \tilde{\imath}_{6},
$$

and so $H\left(\delta \eta_{6} p_{7}\right)=\tilde{\imath}_{6} \eta_{6} p_{7}$.

By use of a generalized version [9] of [20, Proposition 2.2] and by the fact that $H\left(\nu^{\prime}\right)=\eta_{5}[20,(5.3)]$ for $H: \pi_{6}^{3} \rightarrow \pi_{6}^{5}$, we obtain

$$
H\left(\overline{i_{4} \nu^{\prime}}\right) \circ i_{7}=H\left(i_{4} \nu^{\prime}\right)=\left(i_{4} \wedge i_{3}\right) \eta_{5}=\left(i_{4} \wedge i_{3}\right) \bar{\eta}_{5} \circ i_{7} \in \pi_{6}\left(\Sigma M^{3} \wedge M^{3}\right) .
$$

Hence, by use of $(*)_{6}$, we obtain

$$
H\left(\overline{\imath_{4} \nu^{\prime}}\right) \equiv\left(i_{4} \wedge i_{3}\right) \bar{\eta}_{5} \bmod \pi_{7}\left(\Sigma M^{3} \wedge M^{3}\right) \circ p_{7}=\left\{\tilde{\imath}_{6} \eta_{6} p_{7},\left(\iota_{M^{4}} \wedge i_{3}\right) \tilde{\eta}_{5} p_{7}\right\} .
$$

Thus (ii) follows from the fact that $H \circ \Sigma=0$. This completes the proof.

For the cell complex $\mathrm{P}_{3}^{6}=M^{4} \cup_{i_{4} \bar{\eta}_{3}} C M^{5}$, we set

$$
i^{\prime}=i_{M^{4}, \mathrm{P}_{3}^{6}}, \quad i^{\prime \prime}=i^{\prime} \circ i_{4}, \quad p^{\prime}=p_{\mathrm{P}_{3}^{6}, M^{4}} \quad \text { and } \quad p^{\prime \prime}=p_{6} \circ p^{\prime} .
$$

Let $\widetilde{\imath_{5} \eta_{4} p_{5}}$ be a coextension of $i_{5} \eta_{4} p_{5}=2 \iota_{M^{5}}$. It is taken as a representative of the Toda bracket

$$
\left\{i^{\prime}, i_{4} \bar{\eta}_{3}, i_{5} \eta_{4} p_{5}\right\} \subset\left[M^{6}, \mathrm{P}_{3}^{6}\right] .
$$

Then, by the properties of Toda brackets and by the fact that

$$
\left\{2 \iota_{5}, p_{5}, i_{5}\right\} \ni \iota_{5} \bmod 2 \iota_{5},
$$

we see that

$$
\begin{aligned}
\widetilde{2 \imath_{5} \eta_{4} p_{5}} & \in\left\{i^{\prime}, i_{4} \bar{\eta}_{3}, i_{5} \eta_{4} p_{5}\right\} \circ i_{6} \eta_{5} p_{6} \\
& =i^{\prime} \circ\left\{i_{4} \bar{\eta}_{3}, i_{5} \eta_{4} p_{5}, i_{5}\right\} \circ \eta_{5} p_{6} \\
& \supset i^{\prime} \circ\left\{2 \tilde{\eta}_{3}, p_{5}, i_{5}\right\} \circ \eta_{5} p_{6} \\
& \supset i^{\prime} \tilde{\eta}_{3} \circ\left\{2 \iota_{5}, p_{5}, i_{5}\right\} \circ \eta_{5} p_{6} \\
& \ni i^{\prime} \tilde{\eta}_{3} \eta_{5} p_{6} \bmod i_{*}^{\prime}\left[M^{6}, M^{4}\right] \circ 2 \iota_{M^{6}}+\left[M^{6}, \mathrm{P}_{3}^{6}\right] \circ 2 \iota_{M^{6}} \circ 2 \iota_{M^{6}}
\end{aligned}
$$

By (1.3) and Lemma 2.1 (iii), the indeterminacy $i_{*}^{\prime}\left[M^{6}, M^{4}\right] \circ 2 \iota_{M^{6}}+\left[M^{6}, \mathrm{P}_{3}^{6}\right] \circ$ $2 \iota_{M^{6}} \circ 2 \iota_{M^{6}}$ is trivial. That is,

$$
\widetilde{2 \imath_{5} \eta_{4} p_{5}}=i^{\prime} \tilde{\eta}_{3} \eta_{5} p_{6} \in\left[M^{6}, \Sigma \mathrm{P}_{3}^{6}\right] \text {. }
$$

Since $p_{4} \circ i_{4} \bar{\eta}_{3}=0$, there exists an extension $\bar{p}_{4} \in\left[\mathrm{P}_{3}^{6}, S^{4}\right]$ of $p_{4}$. We show the following.

Lemma 4.2. $2 \iota_{\Sigma \mathrm{P}_{3}^{6}} \equiv \pm \Sigma \widetilde{\iota_{5} \eta_{4} p_{5}} \Sigma p^{\prime} \bmod \left(\Sigma i^{\prime \prime}\right) \nu_{4} \Sigma p^{\prime \prime}$.

Proof. By use of the canonical bijection

$$
\left[C \mathrm{P}_{3}^{6}, \mathrm{P}_{3}^{6} ; \Sigma \mathrm{P}_{3}^{6}, M^{5}\right] \cong\left[\Sigma \mathrm{P}_{3}^{6}, M^{7}\right]=\left\{\Sigma p^{\prime}\right\}
$$


we obtain the exact sequence

$$
\left[\Sigma \mathrm{P}_{3}^{6}, M^{5}\right] \stackrel{\left(\Sigma i^{\prime}\right)_{*}}{\longrightarrow}\left[\Sigma \mathrm{P}_{3}^{6}, \Sigma \mathrm{P}_{3}^{6}\right] \stackrel{\left(\Sigma p^{\prime}\right)_{*}}{\longrightarrow}\left[\Sigma \mathrm{P}_{3}^{6}, M^{7}\right] \longrightarrow 0 .
$$

Since $\left(\Sigma p^{\prime}\right)_{*}\left(2 \iota_{\Sigma \mathrm{P}_{3}^{6}}-\Sigma \widetilde{\Sigma_{5} \eta_{4} p_{5}} \Sigma p^{\prime}\right)=0$, we get that

$$
2 \iota_{\Sigma \mathrm{P}_{3}^{6}}-\Sigma \widehat{\iota_{5} \eta_{4} p_{5}} \Sigma p^{\prime} \in\left(\Sigma i^{\prime}\right)_{*}\left[\Sigma \mathrm{P}_{3}^{6}, M^{5}\right] .
$$

Making use of the exact sequence induced from the cofiber sequence starting with $i_{5} \bar{\eta}_{4}: M^{6} \rightarrow M^{5}$ :

$$
\begin{aligned}
& {\left[M^{6}, M^{5}\right] \stackrel{\left(i_{5} \bar{\eta}_{4}\right)^{*}}{\longleftarrow}\left[M^{5}, M^{5}\right] \stackrel{\left(\Sigma i^{\prime}\right)^{*}}{\longleftarrow}\left[\Sigma \mathrm{P}_{3}^{6}, M^{5}\right] } \\
& \stackrel{\left(\Sigma p^{\prime}\right)^{*}}{\longleftarrow}\left[M^{7}, M^{5}\right] \stackrel{\left(i_{6} \bar{\eta}_{5}\right)^{*}}{\longleftarrow}\left[M^{6}, M^{5}\right],
\end{aligned}
$$

together with (1.3) and Lemma 2.1 (i), we obtain

$$
\left[\Sigma \mathrm{P}_{3}^{6}, M^{5}\right]=\left\{i_{5} \eta_{4} \Sigma \bar{p}_{4}, \tilde{\eta}_{4} \eta_{6} \Sigma p^{\prime \prime}, i_{5} \nu_{4} \Sigma p^{\prime \prime}\right\} \cong\left(\mathbb{Z}_{2}\right)^{3} .
$$

So, by the fact that $i^{\prime} \circ i_{4} \eta_{3}=i^{\prime} \circ i_{4} \bar{\eta}_{3} \circ i_{5}=0$ and by (4.1), we obtain

$$
\left(\Sigma i^{\prime}\right)_{*}\left[\Sigma \mathrm{P}_{3}^{6}, M^{5}\right]=\left\{2 \Sigma \widetilde{\imath_{5} \eta_{4} p_{5}} \Sigma p^{\prime},\left(\Sigma i^{\prime \prime}\right) \nu_{4} \Sigma p^{\prime \prime}\right\} .
$$

This leads to the relation, completing the proof.

In fact we can show the following.

Remark 4.3. $\left[\Sigma \mathrm{P}_{3}^{6}, \Sigma \mathrm{P}_{3}^{6}\right]=\mathbb{Z}_{8}\left\{\iota_{\Sigma \mathrm{P}_{3}^{6}}\right\} \oplus \mathbb{Z}_{2}\left\{\left(\Sigma i^{\prime \prime}\right) \nu_{4} \Sigma p^{\prime \prime}\right\}$.

Now we prove Theorem 1.4.

Proof of Theorem 1.4.

We consider the exact sequence $\left(i=\Sigma i_{4,6}, p=\Sigma p_{6,4}\right)$

$$
\left[\Sigma \mathrm{P}^{4}, \Sigma \mathrm{P}^{6}\right] \stackrel{i^{*}}{\longleftarrow}\left[\Sigma \mathrm{P}^{6}, \Sigma \mathrm{P}^{6}\right] \stackrel{p^{*}}{\longleftarrow}\left[M^{7}, \Sigma \mathrm{P}^{6}\right] .
$$

By use of the commutative diagram:

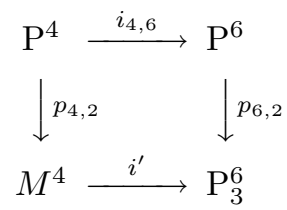

and by (2.7),

$$
\overline{\left(\Sigma i_{1,4}\right) \eta_{2} \bar{\eta}_{3}} \circ \Sigma p_{6,2} \circ \Sigma i_{4,6}=\left(\Sigma i_{1,4}\right) \eta_{2} \bar{\eta}_{3} \Sigma p_{4,2}=4 \iota_{\Sigma \mathrm{P}^{4}} .
$$

So, by the relation

$$
\Sigma i_{4,6} \circ 4 \iota_{\Sigma \mathrm{P}^{4}}=4 \iota_{\Sigma \mathrm{P}^{6}} \circ \Sigma i_{4,6} \in\left[\Sigma \mathrm{P}^{4}, \Sigma \mathrm{P}^{6}\right],
$$


we have Theorem 1.4 (i).

By Lemma 4.2, we see that

$$
\begin{aligned}
2 \overline{\left(\Sigma i_{1,4}\right) \eta_{2} \bar{\eta}_{3}}= & \overline{\left(\Sigma i_{1,4}\right) \eta_{2} \bar{\eta}_{3}} \circ 2 \iota_{\Sigma \mathrm{P}_{3}^{6}} \\
\equiv & \pm \overline{\left(\Sigma i_{1,4}\right) \eta_{2} \bar{\eta}_{3}} \circ \Sigma \widehat{\iota_{5} \eta_{4} p_{5}} \Sigma p^{\prime} \\
& \bmod \overline{\left(\Sigma i_{1,4}\right) \eta_{2} \bar{\eta}_{3}} \circ\left(\Sigma i^{\prime \prime}\right) \nu_{4} \Sigma p^{\prime \prime} .
\end{aligned}
$$

By the relation $\eta_{3} \nu_{4}=\nu^{\prime} \eta_{6}$ and (3.4),

$$
\begin{aligned}
\overline{\left(\Sigma i_{1,4}\right) \eta_{2} \bar{\eta}_{3}} \circ\left(\Sigma i^{\prime \prime}\right) \nu_{4} & =\left(\Sigma i_{1,4}\right) \eta_{2} \eta_{3} \nu_{4} \\
& =\left(\Sigma i_{1,4}\right) \eta_{2} \nu^{\prime} \eta_{6}=0 \in \pi_{7}\left(\Sigma \mathrm{P}^{4}\right) .
\end{aligned}
$$

By (4.1), (2.1) and (3.4),

$$
\begin{aligned}
2\left(\overline{\left(\Sigma i_{1,4}\right) \eta_{2} \bar{\eta}_{3}} \circ \Sigma \widetilde{\varepsilon_{5} \eta_{4} p_{5}}\right) & =\overline{\left(\Sigma i_{1,4}\right) \eta_{2} \bar{\eta}_{3}} \circ\left(\Sigma i^{\prime}\right) \tilde{\eta}_{4} \eta_{6} p_{7} \\
& =\left(\Sigma i_{1,4}\right) \eta_{2} \nu^{\prime} \eta_{6} p_{7}=0 \in\left[M^{7}, \Sigma \mathrm{P}^{4}\right] .
\end{aligned}
$$

Hence we conclude that

$$
2 \overline{\left(\Sigma i_{1,4}\right) \eta_{2} \bar{\eta}_{3}}=\overline{\left(\Sigma i_{1,4}\right) \eta_{2} \bar{\eta}_{3}} \circ \Sigma \widetilde{\imath_{5} \eta_{4} p_{5}} \Sigma p^{\prime} \in\left[\Sigma \mathrm{P}_{3}^{6}, \Sigma \mathrm{P}^{4}\right] .
$$

By [20, Proposition 1.7], we obtain

$$
\begin{aligned}
& \overline{\left(\Sigma i_{1,4}\right) \eta_{2} \bar{\eta}_{3}} \circ \Sigma \widetilde{\imath_{5} \eta_{4} p_{5}} \in\left\{\left(\Sigma i_{1,4}\right) \eta_{2} \bar{\eta}_{3}, i_{5} \bar{\eta}_{4}, i_{6} \eta_{5} p_{6}\right\}_{1} \\
& \bmod \left(\Sigma i_{1,4}\right) \eta_{2} \bar{\eta}_{3} \circ \Sigma\left[M^{6}, M^{4}\right]+\left[M^{7}, \Sigma \mathrm{P}^{4}\right] \circ i_{7} \eta_{6} p_{7} .
\end{aligned}
$$

By (1.3) and Theorem 1.3, $\left[M^{7}, \Sigma \mathrm{P}^{4}\right] \circ i_{7} \eta_{6} p_{7}=2\left[M^{7}, \Sigma \mathrm{P}^{4}\right]=0$. By Lemma 4.1 (i), (2.1), (3.4) and by the relation $\left(\Sigma i_{1,4}\right) \eta_{2}^{3}=0$, we obtain

$$
\left(\Sigma i_{1,4}\right) \eta_{2} \bar{\eta}_{3} \circ \Sigma\left[M^{6}, M^{4}\right]=\left\{\left(\Sigma i_{1,4}\right) \eta_{2}^{3} \bar{\eta}_{5},\left(\Sigma i_{1,4}\right) \eta_{2} \nu^{\prime} \eta_{6} p_{7}\right\}=0 .
$$

Hence the indeterminacy is trivial and we get that

$$
\overline{\left(\Sigma i_{1,4}\right) \eta_{2} \bar{\eta}_{3}} \circ \Sigma \widetilde{\imath_{5} \eta_{4} p_{5}}=\left\{\left(\Sigma i_{1,4}\right) \eta_{2} \bar{\eta}_{3}, i_{5} \bar{\eta}_{4}, i_{6} \eta_{5} p_{6}\right\}_{1} \in\left[M^{7}, \Sigma \mathrm{P}^{4}\right] .
$$

This leads to Theorem 1.4 (ii).

Since $\left(\Sigma p_{5,4}\right)\left(\Sigma \gamma_{5}\right)=2 \iota_{6}$ by $(1.2)$, we obtain

$$
\Sigma \gamma_{5} \in\left\{\Sigma i_{4,5}, \Sigma \gamma_{4}, 2 \iota_{5}\right\}
$$

By the properties of Toda brackets,

$$
\left(\Sigma \gamma_{5}\right) \eta_{6} p_{7} \in\left\{\Sigma i_{4,5}, \Sigma \gamma_{4}, 2 \iota_{5}\right\} \circ \eta_{6} p_{7}=\Sigma i_{4,5} \circ\left\{\Sigma \gamma_{4}, 2 \iota_{5}, \eta_{5} p_{6}\right\} .
$$

The indeterminacy of $\Sigma i_{4,5} \circ\left\{\Sigma \gamma_{4}, 2 \iota_{5}, \eta_{5} p_{6}\right\}$ is $\left(\Sigma i_{4,5} \circ \Sigma \gamma_{4}\right) \circ\left[M^{7}, S^{5}\right]+\Sigma i_{4,5} \circ$ $\pi_{6}\left(\Sigma \mathrm{P}^{4}\right) \circ \eta_{6} p_{7}=0$ by (1.1) and Corollary 3.7. Therefore

$$
\left(\Sigma \gamma_{5}\right) \eta_{6} p_{7}=\Sigma i_{4,5} \circ\left\{\Sigma \gamma_{4}, 2 \iota_{5}, \eta_{5} p_{6}\right\} .
$$


By the fact that $\Sigma\left[M^{6}, S^{4}\right]=\left[M^{7}, S^{5}\right]$,

$$
\left\{\Sigma \gamma_{4}, 2 \iota_{5}, \eta_{5} p_{6}\right\}=\left\{\Sigma \gamma_{4}, 2 \iota_{5}, \eta_{5} p_{6}\right\}_{1},
$$

and hence

$$
\left(\Sigma \gamma_{5}\right) \eta_{6} p_{7}=\Sigma i_{4,5} \circ\left\{\Sigma \gamma_{4}, 2 \iota_{5}, \eta_{5} p_{6}\right\}_{1} .
$$

By (2.6), (1.4), (2.5) and by the relation $i_{4} \eta_{3}^{2}=i_{4} \bar{\eta}_{3} \circ i_{5} \bar{\eta}_{4} \circ i_{6}$, we obtain

$$
\begin{aligned}
\left\{\Sigma \gamma_{4}, 2 \iota_{5}, \eta_{5} p_{6}\right\}_{1} \subset & \left\{\left(\Sigma i_{3,4}\right) h^{\prime \prime}, 2 \tilde{\eta}_{3}, \eta_{5} p_{6}\right\}_{1} \\
= & \left\{\left(\Sigma i_{3,4}\right) h^{\prime \prime}, i_{4} \eta_{3}^{2}, \eta_{5} p_{6}\right\}_{1} \\
\supset & \left\{\left(\Sigma i_{1,4}\right) \eta_{2} \bar{\eta}_{3}, i_{5} \bar{\eta}_{4}, i_{6} \eta_{5} p_{6}\right\}_{1} \\
& \bmod \left(\Sigma i_{3,4}\right) h^{\prime \prime} \circ \Sigma\left[M^{6}, M^{3}\right]+\pi_{6}\left(\Sigma \mathrm{P}^{4}\right) \circ \eta_{6} p_{7} .
\end{aligned}
$$

By Corollary 3.7, $\pi_{6}\left(\Sigma \mathrm{P}^{4}\right) \circ \eta_{6} p_{7}=0$. By Lemma 4.1 (ii) and (2.6),

$$
\left(\Sigma i_{3,4}\right) h^{\prime \prime} \circ \Sigma\left[M^{6}, M^{3}\right]=\left\{\left(\Sigma \gamma_{4}\right) \bar{\eta}_{5}\right\} .
$$

So we obtain

$$
\left\{\Sigma \gamma_{4}, 2 \iota_{5}, \eta_{5} p_{6}\right\}_{1} \equiv\left\{\left(\Sigma i_{1,4}\right) \eta_{2} \bar{\eta}_{3}, i_{5} \bar{\eta}_{4}, i_{6} \eta_{5} p_{6}\right\}_{1} \bmod \left(\Sigma \gamma_{4}\right) \bar{\eta}_{5} .
$$

Thus, by (1.1) and (4.2), we obtain

$$
\left(\Sigma \gamma_{5}\right) \eta_{6} p_{7}=\left(\Sigma i_{4,5}\right) \circ\left\{\left(\Sigma i_{1,4}\right) \eta_{2} \bar{\eta}_{3}, i_{5} \bar{\eta}_{4}, i_{6} \eta_{5} p_{6}\right\}_{1} .
$$

This leads to Theorem 1.4 (iii), completing the proof of Theorem 1.4.

Acknowledgements. The author wishes to thank Morisugi and Iwase for the encouragements during the expedition to the conjecture. The author also wishes to thank Fred Cohen for the cordial hospitality during the stay at Rochester University on March, 2000.

\author{
Department of Mathematical Sciences \\ Faculty of Science, Shinshu University \\ MATSUMOTO 390-8621, JAPAN \\ e-mail: jmukai0@gipac.shinshu-u.ac.jp
}

\title{
References
}

[1] J. F. Adams, Vector fields on spheres, Ann. of Math. 75 (1962), 603-632.

[2] M. Arkowitz, The generalized Whitehead product, Pacific. J. Math. 12 (1962), 7-23. 
[3] M. G. Barratt, Track groups (II), Proc. London Math. Soc. (3) 5 (1955), $285-329$.

[4] _ Spaces of finite characteristic, Quart. J. Math. Oxford Ser. (2) 11 (1960), 124-136.

[5] M. G. Barratt, I. M. James and N. Stein, Whitehead products and projective spaces, J. Math. Mech. 9 (1960), 813-819.

[6] H. J. Baues, Commutator calculus and groups of homotopy classes, London Math. Soc. Lecture Note 50, 1981.

[7] A. L. Blakers and W. S. Massey, The homotopy groups of a triad. II, Ann. of Math. 55 (1952), 192-201.

[8] _ Products in homotopy theory, Ann. of Math. 58 (1953), 295-324.

[9] T. Inoue and J. Mukai, A note on the Hopf homomorphism of a Toda bracket and its application, to appear in Hiroshima Math. J. 33-3 (2003).

[10] I. M. James, On the homotopy groups of certain pairs and triads, Quart. J. Math. Oxford Ser. (2) 5 (1954), 260-270.

[11] K. Morisugi, Hopf constructions, Samelson products and suspension maps, Contemp. Math. 239 (1999), 225-238.

[12] J. Mukai, A note on the Kahn-Priddy map, J. Math. Soc. Japan 40 (1988), $53-63$.

[13] _ A remark on Toda's result about the suspension order of the stunted real projective space, Mem. Fac. Sci. Kyushu Univ. A 42 (1988), $87-94$.

[14] - On the attaching map in the Stiefel manifold of 2-frames, Math. J. Okayama Univ. 33 (1991), 177-188.

[15] _ Note on existence of the unstable Adams map, Kyushu J. Math. 49 (1995), 271-279.

[16] Some homotopy groups of the double suspension of the real projective space $\mathbb{R P}^{6}$, Matemática Contemporânea 13 (1997), 235-249.

[17] - Generators of some homotopy groups of the mod 2 Moore space of dimension 3 or 5, Kyushu J. Math. 55 (2001), 63-73.

[18] _ Self-homotopy of a suspended real 4-projective space, Contemp. Math. 274 (2001), 241-255.

[19] P. Silberbush and J. Ucci, On the suspension order of $\left(\mathbb{R P}^{2 m}\right)^{[k]}$, Proc. Amer. Math. Soc. 126 (1998), 1867-1872. 
[20] H. Toda, Composition methods in homotopy groups of spheres, Ann. of Math. Stud. 49, Princeton, 1962.

[21] $\quad$ Order of the identity class of a suspension space, Ann. of Math. 78 (1963), 300-325. 